\title{
Screening for Gastric Cancer in Japan
}

\author{
Takahiro Kozu ${ }^{1}$, Hiroko Shoda ${ }^{1}$, Yukio Muramatsu ${ }^{1}$, \\ and DAIZO SAITO ${ }^{2}$
}

Summary. Gastric endoscopy has not yet been recommended for organized or population-based cancer screening because at the moment, the sole criterion for evaluating the effectiveness of cancer screening is the reduction in the death rate, and not the mere detection of cancer. Nevertheless, compared with X-ray screening, which has normally been recommended, endoscopic screening is better at finding small lesions, at finding cancer at its earlier stages, making it more easily and economically treatable, and allows on-the-spot biopsies. Opportunistic, individually initiated screening by endoscopy is more and more in demand. Therefore, its excellent efficacy needs to be matched by improved toleration, improved safety, and improved manpower efficiency so that it can be standardized and utilized to its full diagnostic, therapeutic, and quality-of-life potential.

Key words. Gastric cancer, Screening, Endoscopy

\section{Introduction}

In Japan, cancer screening based on monitoring the death from cancer of elderly patients has been managed by the government since 1983, and radiological gastrography (X-ray) is recommended because of its effectiveness in decreasing the rate of death from gastric cancer.However, owing to remarkable progress in endoscopy technology in the past quarter of a century, endoscopy has become the most popular method of examination not only in clinical situations, but also for screening purposes. However, there are some problems which still have to be solved, such as toleration, safety, and manpower, before mass screening by endoscopy can ever be considered. The purpose of this chapter is to consider the present problems and solutions, and search for a standardization that will make mass utilization possible.

\footnotetext{
${ }^{1}$ National Cancer Center, Research Center for Cancer Prevention and Screening, Cancer Screening Division, 5-1-1 Tsukiji, Chuo-ku, Tokyo 104-0045, Japan

${ }^{2}$ National Cancer Center Hospital, Endoscopy Division, Tokyo, Japan
} 


\section{Assessment of Effectiveness}

In Japan, the most common methods of screening for gastric cancer are radiological gastrography (X-ray), pepsinogen assay, Helicobacter pylori antibody count, and endoscopy. An evaluation by a study group from the Ministry of Welfare, in a report entitled "Gastric Cancer Guidelines Based on the Evaluation of Effectiveness" stated that the X-ray is an effective screening method owing to its cohort study result, but that the pepsinogen assay was doubtful, and a Helicobacter pylori antibody count was not effective. The guidelines also reported that "screening endoscopy is not recommended for population-based screening" because there is no evidence that it decreases the cancer death rate, but that it may be used for opportunistic screening, and is available with informed consent when sought by an individual (Table 1).

\section{Screening by Endoscopy}

\section{Advantages}

A nationwide research study (including only institutions which have more than 500 cases per year) into screening endoscopy for gastric cancer reported that 287 gastric cancers were detected out of 93909 , that the detection rate was $0.31 \%$, and that the rate of early gastric cancer cases was $67.9 \%$ (195 cases). On the other hand, the detection rate by X-ray screening was $0.094 \%$ and its ratio of early cancer was $68.4 \%$. The ratio of early cancer was the same, but when the overall cancer detection rate was found to be $0.14 \%$ in subsequent tests, the detection rate by endoscopy was shown to be 3-4 times that by $\mathrm{X}$-ray, which proves that endoscopy is very precise [1] (Table 2).

TABLE 1. Summary of present "recommendations" for gastric cancer screening.

\begin{tabular}{lcl}
\hline & Population-based Screening & Opportunistic Screening \\
\hline X-ray & Recommended & Recommended \\
PG & Not recommended & As decided by individual \\
HP & Not recommended & As decided by individual \\
GF & Not recommended & As decided by individual \\
\hline
\end{tabular}

PG, Pepsinogen; HP, Helicobacter pylori antibody; GF, gastroscopy

TABLE 2. Result of screening by endoscope.

\begin{tabular}{lr}
\hline Number of receivers & \multicolumn{1}{l}{93,909} \\
$\mathrm{M}$ & $50,876(54.2 \%)$ \\
$\mathrm{F}$ & $43,033(45.8 \%)$ \\
Detected lesion and detection rate & \\
Gastric cancer & $287(0.31 \%)$ \\
(Early GC 195) & $(0.21 \%)$ \\
Gastric ulcer & $4,435(4.72 \%)$ \\
Gastric polyp & $7,627(8.12 \%)$ \\
\hline
\end{tabular}

*Detection rate by X-ray screening: $0.094 \%$ (GC) 


\section{High Precision in Diagnosis}

An endoscopy examination used to be the second test to check the details of a lesion after X-ray detection and to make a final diagnosis, but based on nationwide endoscopy screening research, endoscopy is now known to be the most precise method of checking the gastrointestinal tract.

\section{Biopsy}

When abnormal findings are detected, a histological diagnosis from a biopsy specimen is helpful whenever it is difficult to make a diagnosis from endoscopic findings.

\section{Subjective Organs}

Not only the stomach, but also the esophagus and duodenum, can easily be observed by panendoscopy.

\section{Selection of Subjects}

Of course the subjects screened should be in the high-risk age group, but as there is no possibility of exposure to radiation, younger subjects are not contraindicated. Even subjects who have paralysis, difficulty in hearing, or any other physical handicap can be examined easily because they do not have to change position during the examination.

\section{Problems}

\section{Toleration}

The level of technique varies widely depending on the examiner, even with a thin scope or sedation. The trend for transnasal endoscopy has shown that the most difficult part is insertion into the esophagus from the throat. Even when the examination has been completed very smoothly with deep sedation, over-inflation can cause abdominal fullness or nausea afterwards. Therefore examiners have to have a good technique so that the patient is comfortable even after the examination is finished.

\section{Complications}

An endoscopy examination has a few risks, such as shock from the preparation medicine, perforation, or infection. Research by the Japan Gastroenterological Endoscopy Society (1998-2002) reported that the complication rate was $0.12 \%(997 / 826313)$ with panendoscopy. The death rate was $0.0076 \%$ (63/826313), and 19 cases died from the observation examination only (without biopsy or therapeutic endoscopy). The complication rate due to preparation was $0.0059 \%$ (754/12 844551), and the death rate was $0.00011 \%$ (14/12 844551). The frequency was highest in cases with anti-bowel-movement medicine or sedation [2] (Table 3). 
TABLE. 3. Complications of Endoscopie examinations for recently 5 years.

\begin{tabular}{lc}
\hline Whole (including ERCP, CS) & \multicolumn{1}{c}{ Pan-endoscope } \\
\hline Total No.: 12,844,551 & Total No.: 8,263,813 \\
Complicaitons: 4,412 (0.032\%) & Complicaiton: $997(0.012 \%)$ \\
By preparation & Death: $63(0.00076 \%)$ \\
Complicaitons: 754 & Observation: 19 \\
Death: 14 & Biopsy: 1 \\
Local anesthesia: 1 & Stop bleeding: 10 \\
Mixed: 6 & Polypectomy, EMR: 4 \\
Sedation only: 2 & Varices therapy: 16 \\
Analgesic drug: 2 & PEG: 8 \\
others: 3 & Others: 5 \\
\hline
\end{tabular}

Japan Gastroenterological Endoscopy Society (1998-2002)

\section{Infection}

Infections thought to be related to the use of endoscopy are reported to be hepatitis B virus (HBV) (1 case), hepatitis C virus (HCV) (1 case), H. pylori (2 cases), blood poisoning (1 case), and one other, but it is nearly impossible to confirm direct cause and effect. Medical accidents totaled 144 cases, and glutalaldehyde-dependent eye and skin diseases, as well as asthma, are rather common. HBV infections totaled 3 cases, and HCV infections totaled 8 cases [2].

\section{Omissions}

Yoshida et al. [3] have presumed that when there is an outbreak within 3 years of an endoscopic examination it will be a false negative, and have retrospectively found a rate of $25.6 \%$ ( 62 cases out of 242). It has also been reported that the majority of those cases $(58$ lesions $=83.9 \%$ ) were unnoticed during the endoscopy, while 10 lesions $(14.7 \%)$ were unnoticed in a biopsy. Hosokawa et al. [4] examined stomach cancer detection performance retrospectively for 3 years, and reported finding $18.3 \%$ of false-negative examinations $(150 / 820)$. In order to decrease the number of false-negative examinations, it is suggested that both examination technique and observation accuracy need to be increased by means of further practice and also by a more positive use of biopsies.

\section{Manpower}

An endoscope examination needs at least $5 \mathrm{~min}$ for adequate observation, image taking, and evaluation. The examination time is not very different from that needed for a radiographic examination, but the preliminary questionnaire, the set-up, and the post-examination rest all require specialized staff. If the skill of all examiners is considered to be equal, the processing capacity is proportional to the number of examining physicians, but compared with radiologists, the number of endoscopists, especially those engaged in screening, is clearly much smaller, and the present situation is thought likely to continue. In short, it is impossible to increase the number of 
TABLE 4. Conditions necessary for screening method.

- show evidence of decreasing the mortality rate by cancers

- be safe

- be easy for receiver \& medical staffs

- be less expensive (than treatments necessitated by lack of screening)

- be less labor intensive

- have higher through put for processing efficiently

screening physicians to any considerable extent, so the development of time-saving disinfection devices and solutions, as well as efficient observation recording devices and experienced, safety-minded staff, are thought to be the key to solving this problem.

\section{Toward Standardization}

At present, procedures are not fully standardized and the methods applied depend on the institution; sedation, endoscope make and model, and observation protocol may vary, resulting in uneven performances. However, in order that more people use endoscopy for check-ups, the toleration must be better, the risks must be lower, and detection reliability must be higher. Therefore, the Standardization Group of the Gastroscopic Digestive Tract Cancer Detection Study Group is discussing the following ways of improving: (1) procedures (informed consent, diagnostic questionnaire and protocol, preparation, scanning protocol and slice display, coloration, pathological diagnosis); (2) how to reduce errors and increase accuracy (doublecheck, scheduling, rating examiners, etc.); (3) classification of subjects according to risk; (4) minimization of risk from examination (including preparation and sedation, etc.). The group thereby aims to provide detailed methods for improving the recommendation rate for endoscopic examination (Table 4).

\section{Conclusion}

The present state and problems of gastroendoscopy are listed above. It is clear that the procedure is very effective, and is beginning to be used more and more as a first choice screening method. There is no more reliable visualization method if the stomach needs to be removed or if a posttreatment assessment is required. However, even more important is the fact that with advanced surgical techniques, early detection can result in cure with minimum invasiveness and almost no loss in quality of life. Therefore, for those individuals who would prefer periodic check-ups, this method can meet their demand effectively. In addition, thanks to the tireless efforts of many medical practitioners, safety and comfort have been greatly improved. Now, our goal is to standardize procedures so that the same high level of quality is available everywhere. In addition, in the near future, many new features such as narrow-band imaging (NBI), autofluorescence imaging (AFI), swallowable endoscopic capsules, virtual endoscopy, and zoom lens endoscopy will become available. 


\section{References}

1. All Japan Statistical Committee (2007) The nationwide research of gastroenterological cancer screening in 2004 (in Japanese). J Gastroenterol Cancer Screening 45:49-68

2. Kaneko E, Harada H, Kasugai T, The Complication Measures Committee, et al. (2004) The 4th nationwide research about complication in gastroenterological endoscopy 1998-2002 (in Japanese). Gastroenterol Endosc 46:54-61

3. Yoshida T, Baba Y, Maruyama M, et al. (2006) Outcome assessment of endoscopic diagnosis of gastric cancer and adenoma by using a forward-viewing type endoscope. Search for the possibility of implementing endoscopic mass screening for gastric cancer (in Japanese with English abstract). J Gastroenterol Cancer Screening 44:605-615

4. Hosokawa O, Tsuda S, Kidani E, et al. (1998) Diagnosis of gastric cancer up to three years after negative upper gastrointestinal endoscopy. Endoscopy 30:721-723 